\title{
Comparison of effects of seven treatment methods for distal radius fracture on minimizing complex regional pain syndrome
}

\author{
Jian-Hang Wang, Tao Sun
}

Department of Orthopaedics, Yantaishan Hospital, Yantai, China

Submitted: 14 January 2015

Accepted: 27 February 2015

Arch Med Sci 2017; 13, 1: 163-173

DOI: 10.5114 /aoms.2016.59794

Copyright $@ 2016$ Termedia \& Banach

\section{Abstract}

Introduction: Using network meta-analysis, we evaluated the adverse effects of the seven most common treatment methods, i.e., bridging external fixation, non-bridging external fixation, $\mathrm{K}$-wire fixation, plaster fixation, dorsal plating, volar plating, and dorsal and volar plating, by their associated risk of developing complex regional pain syndrome (CRPS) in distal radius fracture (DRF) patients.

Material and methods: Following an exhaustive search of scientific literature databases for high quality studies, randomized controlled trials (RCTs) related to our study topic were screened and selected based on stringent predefined inclusion and exclusion criteria. Data extracted from the selected studies were used for statistical analyses using Stata 12.0 software.

Results: A total of 17 RCTs, including 1658 DRF patients, were enrolled in this network meta-analysis. Among the 1658 DRF patients, 452 received bridging external fixation, 525 received non-bridging external fixation, 154 received K-wire fixation, 84 received plaster fixation, 132 received dorsal plating, 123 received volar plating, and 188 received dorsal and volar plating. When compared to bridging external fixation patients, there was no marked difference in the CRPS risk in DRF patients receiving different treatments (all $p>0.05)$. However, the surface under the cumulative ranking curves (SUCRA) for plaster fixation (77.0\%) and non-bridging external fixation $(71.3 \%)$ were significantly higher compared with the other five methods.

Conclusions: Our findings suggest that compared with bridging external fixation, K-wire fixation, dorsal plating, volar plating, dorsal and volar plating, plaster fixation and non-bridging external fixation might be the better treatment methods to reduce the risk of CRPS in DRF patients.

Key words: distal radius fracture, plaster fixation, dorsal and volar plating, randomized controlled trials, complex regional pain syndrome, network meta-analysis, treatment relative ranking of predictive probabilities.

\section{Introduction}

Distal radius fractures (DRFs) are the most frequent fractures in upper extremities and are a serious public health concern, especially in the older age groups [1]. The underlying injury is frequently associated with falling from a standing height; therefore, slippery conditions are a major risk factor, and it is also seen in high-impact or trauma-related injuries [2]. DRFs are endemic in the Medicare population, and over 85,000 beneficiaries sustain DRFs each year, which is a significant burden in

\author{
Corresponding author: \\ Jian-Hang Wang \\ Department of Orthopaedics \\ Yantaishan Hospital \\ Jiefang Road No. 91 \\ Zhifu District \\ 264000 Yantai, China \\ Phone/fax: \\ +86-0535-6602180 \\ E-mail: \\ wangjianhang629@163.com
}


healthcare costs and in patient care [3]. Etiologically, the reasons are likely multifactorial, and the incidence of DRFs is steadily increasing in the US and many other countries around the world, mostly attributed to aging populations [4]. Known risk factors for DRFs include previous low-energy fracture and a family history of fracture [5]. The peak age for trauma differs between men and women, with men sustaining high-energy injuries around a peak age of 30 years, while in women postmenopausal changes increase the incidence of injuries, irrespective of the height of the fall [6]. Complex regional pain syndrome (CRPS) is a devastating condition, commonly encountered after DRFs. Its incidence is significantly high, and varies between $10.5 \%$ and $37 \%$ [7]. Complex regional pain syndrome is characterized by pain combined with autonomic, sensory, trophic, as well as motor abnormalities [8]. Further, abnormal sensory, motor, sudomotor, vasomotor, and trophic changes are mandatory diagnostic criteria [9]. The goal of DRF treatment is to restore the affected upper extremity within the acceptable mobility and durability requirements [10]. Most DRF treatments are associated with some known risks including the occurrence of CRPS, especially invasive surgical treatments [11].

Bridging external fixation of the wrist for DRF is performed to maintain reduction, but it may result in the loss of palmar tilt [12]. Non-bridging external fixation is directly applied to fix the distal and proximal fragments together, based on closed fracture reduction, followed by employment of an external fixator [13]. It may better accelerate fracture reduction and secure fixation, facilitate functional recovery and ameliorate wrist motion [14]. K-wires are relatively cheap and have many-sided applications. Insertion of $\mathrm{K}$-wires is a common surgical technique for bone stabilization in the hand and wrist [15]. It is a minimally invasive procedure and is associated with good patient outcomes [6]. Plaster fixation is typically used in stable fractures, and braking after fracture operation, and is cheap, easy to use, non-invasive, and easy to achieve the treatment principles of three-point suspension [16]. Dorsal fixation allows direct exposure and reconstruction of the joint by a capsular incision, but requires dissection of the extensor retinaculum and subsequent plate positioning beneath the tendon, which often leads to tendonitis or tendon rupture [17]. Volar plating is designed to allow direct reduction, solid fixation and early mobilization of wrist fractures. The construction of a fixed angle provides a strong fixation in osteoporotic bone and comminuted fractures [18]. It exploits the anatomy of the volar distal radius and aims to avoid the soft tissue complications observed with dorsal plates [18]. A number of studies have shown that bridging external fixation, non-bridging external fixation, K-wire fixation, plaster fixation, dorsal plating, volar plating and dorsal and volar plating have high efficacy for DRF treatment [12, 17, 19]. However, there have been several attempts to rank the seven treatment strategies, and disagreements exist on their efficacy in treatment of DRF $[11,15,20]$.

Compared to traditional meta-analysis, network meta-analysis that uses a common comparator in the abscence of two direct comparisons and gives acess to relevant direct and indirect comparisons to rank several interventions simultaneously $[21,22]$. In view of the advantages of network analysis, we conducted this network meta-analysis aiming to assess and rank the therapies of bridging external fixation, non-bridging external fixation, K-wire fixation, plaster fixation, dorsal plating, volar plating and dorsal and volar plating in reducing CRPS risk in DRF patients.

\section{Material and methods}

\section{Search methods for identification of articles}

We searched PubMed, EBSCO, Ovid, Springerlink, Wiley, Web of Science and Cochrane Library for relevant studies published prior to October, 2014. Studies published in Chinese or English languages were retrieved using a combination of keywords and subject terms. We also manually searched the bibliographies of relevant literature to identify additional relevant studies. The following subject terms and key words were used to maximize the search specificity and sensitivity: distal radius fracture, complex regional pain syndrome, external fixation, internal fixation, bridging external fixation, non-bridging external fixation, K-wire fixation, plaster fixation, dorsal plating, volar plating and dorsal and volar plating.

\section{Study selection}

Studies published in Chinese and English languages were selected in this network meta-analysis if they met criteria as follows: (1) study types were randomized controlled trials (RCTs); (2) interventions were bridging external fixation, non-bridging external fixation, K-wire fixation, plaster fixation, dorsal plating, volar plating or dorsal and volar plating; (3) research subjects were confirmed clinically and by imaging as DRF patients; (4) end outcome was incidence of CRPS. The exclusion criteria were: (1) non-RCT; (2) repeatedly published studies; (3) lack of data integrity; (4) combined use of interventions. 
Data extraction and methodological quality of included studies

Two investigators extracted the data independently, based on a predefined data collection table. The main extracted information included first author, publication year, country, ethnicity, language, disease, age, gender, and number of cases. Disagreements on data extractions were resolved by discussion.

Methodological quality of included RCTs was evaluated by two or more investigators using Cochrane Collaboration's tool to assess risk of bias [23]. The risk of bias covers six domains, including allocation concealment, random sequence generation, blinding of participants or blinding outcome evaluation, selective reporting and other bias, and incomplete outcome data. The detailed assessment criteria were: (1) whether the allocation sequence is properly generated; (2) whether the method applied to conceal the allocation sequence is appropriate; (3) whether the intended blinding is effective; (4) whether the incomplete outcome data are appropriately dealt with; (5) how selective outcome reporting is examined and what is found; (6) whether any other important concerns about bias are covered in the other domains in the tool.

\section{Statistical analysis}

We conducted all statistical analyses with Stata software, version 12.0 (Stata Corporation, College Station, Texas, USA). Odds ratio (OR) with $95 \%$ confidence intervals $(95 \% \mathrm{Cl})$ was evaluated using a fixed-effects model or a random-effects model to assess the influence of intervention group and control group on CRPS of DRF patients. The $Z$ test was applied to measure the pooled effect size [24]. Heterogeneity across enrolled studies was assessed using Cochran $\mathrm{Q}$ and $l^{2}$ statistics $[25,26]$. A random-effects model was used when there was great heterogeneity $\left(I^{2}>50 \%\right)$; otherwise, a fixed-effects model was performed. Network meta-analysis analyzes data from a network of studies involving three or more interventions. The integration between direct evidence obtained from trials directly comparing interventions and indirect evidence that provided information about two treatments derived by a common comparator enhances the accuracy in evaluation and ranks all the treatments for the studied outcome [27]. In each closed loop, we use the inconsistency factor (IF) to evaluate heterogeneity across studies. If the $95 \% \mathrm{Cl}$ of IF values are truncated at zero, it indicates unimportance of the direction of the IF [28]. Funnel plots, which reflect the presence of small-study effects, further provide validation for the reliability of the final results [29]. The assump- tion of consistency models allows the presence of heterogeneity of the intervention effects among studies while no significant differences in study design exist. After the generation of the heterogeneity matrix, the frequentist method was used for the fitted model to calculate the ranking probabilities [30]. We used two ways to rank the treatments. First, posterior probabilities of outcomes were used to calculate probabilities of treatment ranking. Second, the surface under the cumulative ranking probabilities (SUCRA) was used to identify which treatment was the best one.

\section{Results}

\section{Trial characteristics}

The electronic database search initially retrieved 1218 studies, of which 17 studies [14, 3146] met the inclusion criteria for the current network meta-analysis. The studies were eliminated for being duplicates $(n=225)$, letters, reviews, or meta-analyses $(n=29)$, not human studies ( $n=13)$, not related to DRF $(n=273)$, not RCT $(n=219)$, studies not relevant to CRPS $(n=306)$, not relevant to network $(n=129)$, without data $(n=4)$ and studies that had insufficient information or weakly correlated data $(n=3)$. The remaining 17 cohort studies, published between 1996 and 2012, were selected for meta-analysis and contained a combined total of 1658 DRF patients (452 bridging external fixation, 525 non-bridging external fixation, $154 \mathrm{~K}$-wire fixation, 84 plaster fixation, 132 dorsal plating, 123 volar plating, 188 dorsal and volar plating). The flow diagram describes the results of the electronic search. Of the 17 trials, 2 trials were performed in Asians and 15 trials were performed in Caucasians. Additionally, all included studies were two-arm trials and had a total of $17 \mathrm{com}$ parisons. The baseline characteristics and Cochrane assessment of risk of bias are shown in Table I and Figure 1, respectively.

\section{Evidence network}

Figure 2 shows the network of clinical trials based on the comparisons of the seven specific treatment methods, as well as point estimates for the results of traditional meta-analyses. Connecting lines represent direct comparison between the two connected interventions, and pairs of interventions without connection can be compared indirectly by network meta-analysis. The width of lines corresponds to the number of trials, and the size of the node corresponds to the overall sample size of intervention. The color of lines represents the risk of bias of enrolled trials. This study contained seven treatment methods including bridging external fixation, non-bridging external 


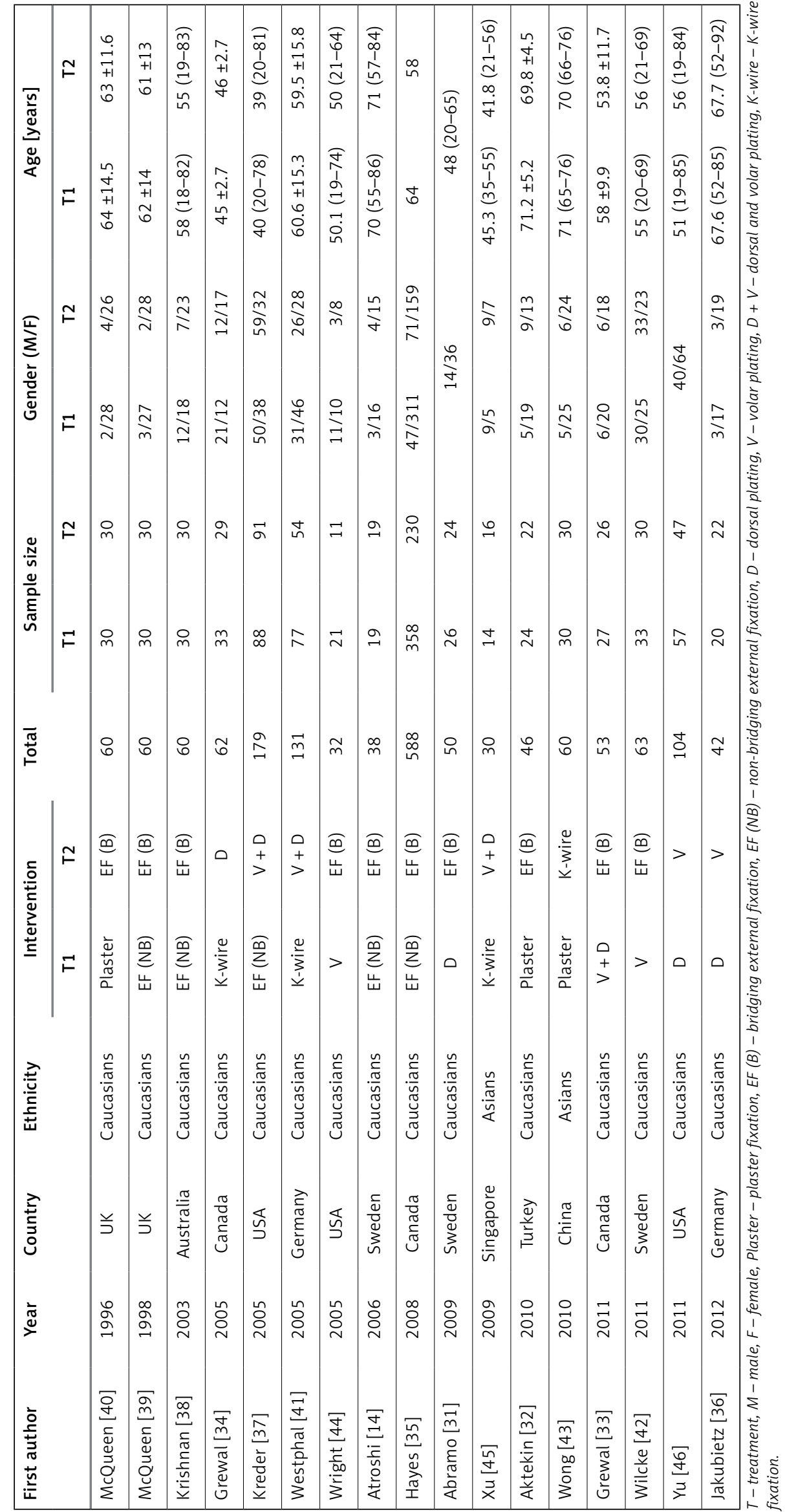




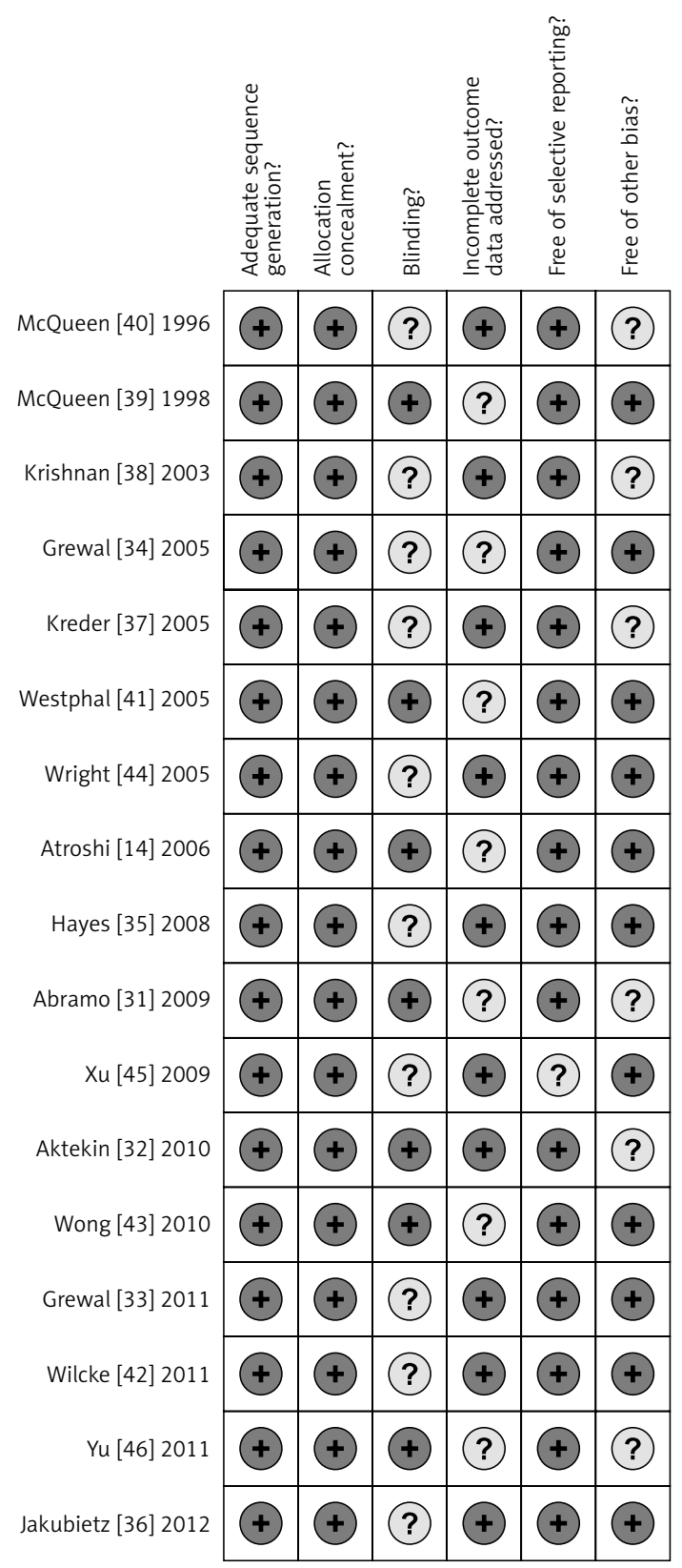

Figure 1. Risk of bias in selected randomized controlled trials (RCTs) using Cochrane Collaboration's tool

fixation, K-wire fixation, plaster fixation, dorsal plating, volar plating and dorsal and volar plating.

\section{Contribution plot}

Each direct comparison contributes differently to the estimation of this network's overall effects, and the details are shown in Figure 3: (1) 4 trials directly compared bridging external fixation and non-bridging external fixation, whose percentage contribution to non-bridging external fixation vs. plaster fixation, non-bridging external fixation vs. dorsal plating, non-bridging external fixation vs. volar plating, and non-bridging external fixation vs.

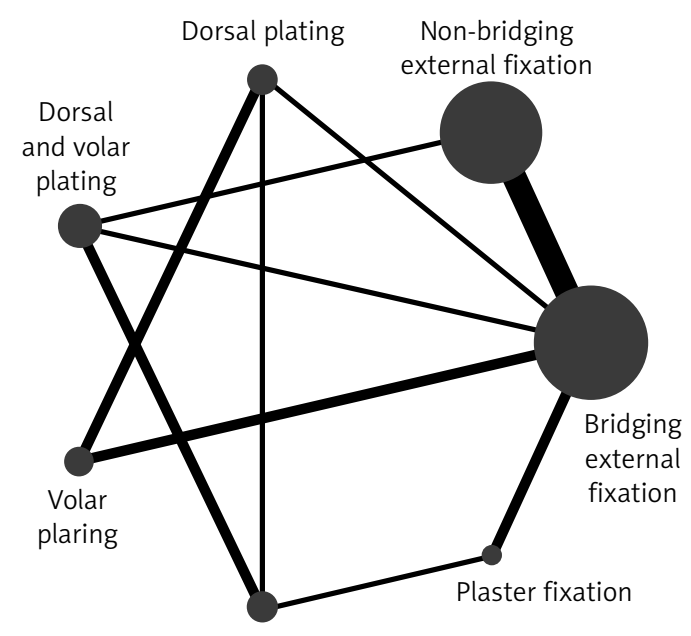

K-wire fixation

Figure 2. The evidence network for trials enrolled in this network meta-analysis

dorsal and volar plating was 50\%, 50\%, 50\%, 50\% and $50 \%$, respectively, $25 \%$ for the overall network meta-analysis; (2) two studies directly compared bridging external fixation and plaster fixation, whose percentage contribution to non-bridging external fixation vs. plaster fixation, plaster fixation vs. dorsal plating, plaster fixation vs. volar plating, and plaster fixation, dorsal and volar plating was $50 \%, 50 \%, 50 \%, 50 \%$ and $50 \%$, respectively, $25 \%$ for the overall network meta-analysis; (3) one trial directly investigated bridging external fixation vs. dorsal plating, whose percentage contribution to non-bridging external fixation vs. dorsal plating, plaster fixation vs. dorsal plating, dorsal plating vs. volar plating, and dorsal plating vs. dorsal and volar plating was 50\%, 50\%, 50\%, $50 \%$ and $50 \%$, respectively, $20 \%$ for the overall network meta-analysis; (4) two studies directly explored bridging external fixation vs. volar plating, whose percentage contribution to non-bridging external fixation vs. volar plating, plaster fixation vs. volar plating, dorsal plating vs. volar plating, and volar plating vs. dorsal and volar plating were $50 \%, 50 \%, 50 \%, 50 \%$ and 50\%, respectively, 20\% for the overall network meta-analysis; (5) one trial studied bridging external fixation vs. dorsal and volar plating, whose percentage contribution to non-bridging external fixation vs. dorsal and volar plating, plaster fixation vs. dorsal and volar plating, dorsal plating vs. dorsal and volar plating, and volar plating vs. dorsal and volar plating were $50 \%, 50 \%, 50 \%, 50 \%$ and $50 \%$, respectively, $20 \%$ for the overall network meta-analysis.

\section{Evaluating and presenting assumptions of network meta-analysis}

An inconsistency plot was applied to test the heterogeneity across studies in the closed loop of this network meta-analysis (Figure 4). This net- 


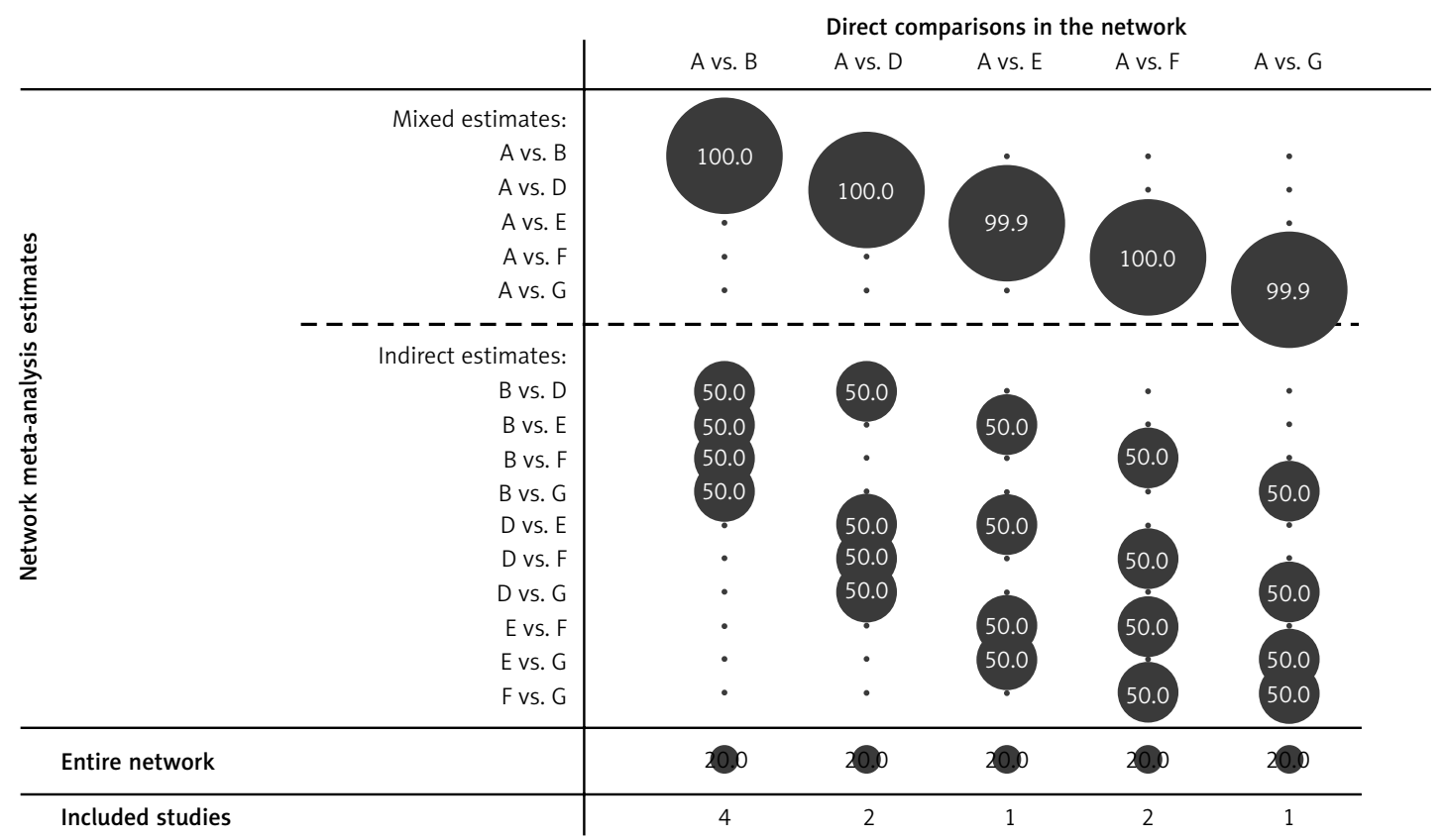

Figure 3. Contribution plot of studies included in this network meta-analysis

A - bridging external fixation, $B$ - non-bridging external fixation, $D$ - plaster fixation, $E$ - dorsal plating, $F$ - volar plating, $G$ - dorsal and volar plating.

\begin{tabular}{|c|c|c|c|}
\hline Loop & IF & $\begin{array}{c}95 \% \mathrm{Cl} \\
\text { (truncated) }\end{array}$ & $\begin{array}{c}\text { Loop-specific } \\
\text { Heterogeneity }\left(\tau^{2}\right)\end{array}$ \\
\hline$A-B-G$ & 0.88 & $(0.00-4.57)$ & 0.000 \\
\hline$A-C-D-G$ & 0.83 & $(0.00-5.44)$ & 0.000 \\
\hline$A-E-F$ & 0.54 & $(0.00-3.84)$ & 0.000 \\
\hline$A-C-E-G$ & 0.45 & $(0.00-5.30)$ & 0.000 \\
\hline \multirow[t]{6}{*}{$A-C-D-E$} & 0.38 & $(0.00-5.07)$ & 0.000 \\
\hline & & & A-B-G: $Z$ test $(Z=0.471, p=0.638)$ \\
\hline & & & A-C-D-G: $Z$ test $(Z=0.355, p=0.723)$ \\
\hline & & & A-E-F: $Z$ test $(Z=0.323, p=0.747)$ \\
\hline & & & $\mathrm{A}-\mathrm{C}-\mathrm{E}-\mathrm{G}: Z$ test $(Z=0.183, p=0.855)$ \\
\hline & & & A-C-D-G: $Z$ test $(Z=0.160, p=0.873)$ \\
\hline
\end{tabular}

Figure 4. Inconsistency test for direct and indirect comparisons

$A$ - bridging external fixation, $B$ - non-bridging external fixation, $C-K$-wire fixation, $D$ - plaster fixation, $E$ - dorsal plating,

$F$ - volar plating, $G$-dorsal and volar plating.

work meta-analysis consists of two closed-loop triangles and 3 closed-loop quadrilaterals, including the bridging external fixation-non-bridging external fixation-dorsal and volar plating loop, the bridging external fixation-K-wire fixation-plaster fixation-dorsal and volar plating loop, the bridging external fixation-dorsal plating-volar plating loop, the bridging external fixation-K-wire fixation-dorsal plating-dorsal and volar plating loop, and the bridging external fixation-K-wire fixation-plaster fixation-dorsal plating loop. The $95 \% \mathrm{Cl}$ of IF val- ues are truncated at zero, suggesting that there is no significant inconsistency. The $p$-values of these closed loops (all $p>0.05$ ) further confirmed that the direct comparisons together with indirect comparisons of the five closed loops have consistency.

\section{Comparisons of efficacy of the seven treatments for DRF}

Network meta-analysis results demonstrated that there was no significant difference in the risk of CRPS between DRF patients receiving 
Table II. Comparisons of the complex regional pain syndrome in distal radius fracture patients treated with 7 different treatments

\begin{tabular}{|lcccccccc|}
\hline \multirow{2}{*}{ Comparisons } & \multicolumn{3}{c}{ CRPS (correlation not ignored) } & \multicolumn{4}{c|}{ CRPS (correlation ignored) } \\
\cline { 2 - 4 } & OR & \multicolumn{2}{c}{$95 \%$ Cl } & $Z$ & OR & \multicolumn{2}{c|}{$95 \%$ Cl } & $Z$ \\
\cline { 2 - 4 } & & LL & UL & & & LL & UL & \\
\hline EF (NB) vs. EF (B) & 0.762 & 0.420 & 1.383 & -0.89 & 0.763 & 0.419 & 1.387 & -0.89 \\
\hline K-wire vs. EF (B) & 2.097 & 0.299 & 14.689 & 0.75 & 2.133 & 0.159 & 28.440 & 0.57 \\
\hline Plaster vs. EF (B) & 0.609 & 0.178 & 2.082 & -0.79 & 0.609 & 0.178 & 2.082 & -0.79 \\
\hline D vs. EF (B) & 1.143 & 0.258 & 5.060 & 0.18 & 0.439 & 0.037 & 5.175 & -0.65 \\
\hline V vs. EF (B) & 1.209 & 0.327 & 4.469 & 0.28 & 1.726 & 0.378 & 7.882 & 0.70 \\
\hline D + V vs. EF (B) & 1.631 & 0.327 & 8.138 & 0.60 & 1.644 & 0.269 & 10.063 & 0.54 \\
\hline
\end{tabular}

CRPS - complex regional pain syndrome, OR - odds ratio, 95\% Cl - 95\% confidential intervals, EF (NB) - non-bridging external fixation, $E F(B)$ - bridging external fixation, $K$-wire $-K$-wire fixation, Plaster - Plaster fixation, $D$ - dorsal plating, $V-$ volar plating, $D+V-$ dorsal and volar plating.

bridging external fixation and those receiving non-bridging external fixation, K-wire fixation, plaster fixation, dorsal plating and volar plating (non-bridging external fixation: $\mathrm{OR}=0.762$, $95 \% \mathrm{Cl}=0.420-1.383, p=0.372$; K-wire fixation: $\mathrm{OR}=2.097,95 \% \mathrm{Cl}=0.299-14.689, p=0.456$ plaster fixation: $\mathrm{OR}=0.609,95 \% \mathrm{Cl}=0.178-$ 2.082, $p=0.429$; dorsal plating: $\mathrm{OR}=1.143,95 \%$ $\mathrm{Cl}=0.258-5.060, p=0.860$; volar plating: $\mathrm{OR}=$ $1.209,95 \% \mathrm{Cl}=0.327-4.469, p=0.776$; dorsal and volar plating: $\mathrm{OR}=1.631,95 \% \mathrm{Cl}=0.327-8.138$, $p=0.551$ ). After ignoring covariance, further analysis suggests no significant differences among results (Table II).

\section{Ranking of interventions}

The treatment relative ranking of estimated probabilities of the seven treatments for DRF patients were: $51.5 \%$ (bridging external fixation), $71.4 \%$ (non-bridging external fixation), $24.5 \%$ (K-wire fixation), $76.5 \%$ (plaster fixation), $48.3 \%$ (dorsal plating), $44.5 \%$ (volar plating) and 33.2\% (dorsal and volar plating). The SUCRA values of the seven treatments' relative ranking of predictive probabilities were $51.5 \%$ for bridging external fixation, $71.3 \%$ for non-bridging external fixation, $24.5 \%$ for K-wire fixation, $77.0 \%$ for plaster fixation, $48.9 \%$ for dorsal plating, $43.7 \%$ for volar
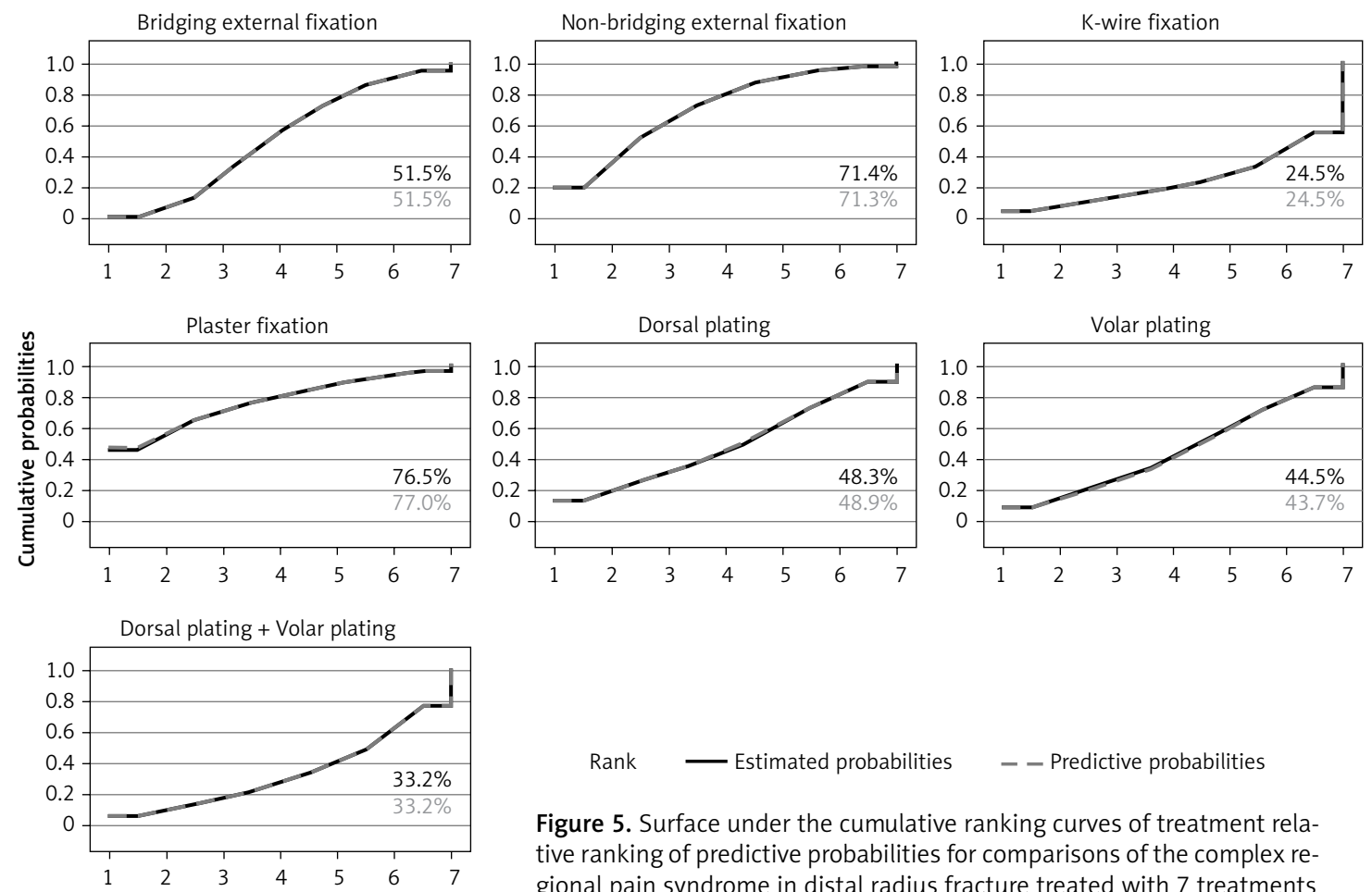

$$
\text { Rank — Estimated probabilities - - Predictive probabilities }
$$

Figure 5. Surface under the cumulative ranking curves of treatment relative ranking of predictive probabilities for comparisons of the complex regional pain syndrome in distal radius fracture treated with 7 treatments 
plating and $33.2 \%$ for dorsal and volar plating, suggesting that plaster fixation and non-bridging external fixation might be the optimum treatments for DRF for the lowest occurrence of CRPS, compared with bridging external fixation, K-wire fixation, dorsal plating, volar plating and dorsal and volar plating (Figure 5).

\section{Assessment of publication bias}

Figure 6 presents the funnel plot for the seven interventions network which provides an indication for the presence of a small-study effect. All the included studies are symmetrically distributed around the vertical line $(x=0)$, suggesting no evidence of a small-study effect in the network.

\section{Discussion}

Distal radius fracture are the most common pediatric orthopedic injuries, and their incidence has increased over the last three decades. A majority of distal radius fractures can be treated successfully with a closed reduction and plaster immobilization alone. In this network meta-analysis, we aimed to rank the seven available treatment modalities, including bridging external fixation, non-bridging external fixation, K-wire fixation, plaster fixation, dorsal plating, volar plating, and dorsal and volar plating, for their associated complication in treatment of DRFs. In this setting, we found no marked differences in CRPS risk between patients treated with bridging external fixation, non-bridging external fixation, K-wire fixation, plaster fixation, dorsal plating, volar plating, and dorsal and volar plating. The SUCRA values of plaster fixation and non-bridging external fixation were, however, significantly higher compared with the other five modalities, suggesting that plaster fixation and

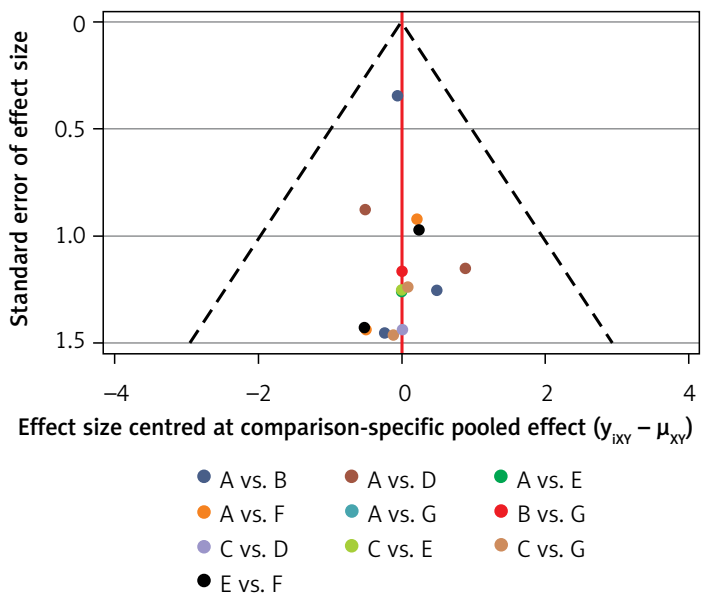

Figure 6. Funnel plots assessment of publication bias of all included studies

$A$ - bridging external fixation, $B$ - non-bridging external fixation, $C-K$-wire fixation, $D$ - plaster fixation, $E$-dorsal plating, $F$-volar plating, $G$-dorsal and volar plating. non-bridging external fixation might be better choices in treatment of DRF, to reduce the incidence of CRPS. SUCRA is a simple transformation of mean rank and is applied to supply a hierarchy of treatments. Greater SUCRA values indicate higher rank of the treatment [47]. As we know, plaster fixation is the first-line treatment for DRFs because it facilitates retention of the reduced position. For instance, it can provide a better fixation effect compared to an orthosis. A plaster cast could be split and spread to relieve pressure by $65 \%$, and thereby it has been a well-established modality, allowing for expansion of the soft tissues after trauma or injury [48]. Splitting the cast ensured the structural stability to be maintained while lowering the CRPS risk from swelling via reducing pressure [48]. This method is cheap, easy to use, non-invasive, and easy to achieve the treatment principle of threepoint suspension, and its fixed effect is excellent, making it a practical approach of external fixation [16]. The main disadvantage of plaster fixation is that it can cause various complications, including compartment syndrome, pressure ulcers, infection, dermatitis, joint contracture, and nerve damage [49]. A poorly or incorrectly applied plaster can result in failure of immobilization resulting in ongoing pain and deformity, pressure necrosis of the skin, compartment syndrome, stiffness, thermal injury and nerve palsy. Nevertheless, a well-applied plaster can provide safe and comfortable immobilization of a fracture prior to operative management or as part of definitive management of an injury. Non-bridging external fixation for DRFs was introduced as a versatile tool to combine closed reduction and faster functional recovery after treatment [19]. The non-bridging external fixator is indicated in extra-articular, dorsally displaced fractures, and has the advantage of reducing the fracture, allowing direct movement of the distal fragment through using the pins as a joystick, and enabling early movement of the wrist [13]. In line with our findings in this meta-analysis, a study by Windolf et al. suggested that non-bridging external fixation provided sufficient stabilization in restoring and retaining anatomy after most DRFs [19]. Potestio et al. also investigated the effectiveness in 56 DRF patients who received non-bridging external fixation, and all patients showed excellent or good clinical outcomes [50].

Additionally, various risk factors including age and gender, as well as time/onset of pain (pre/ post-operative or fixation), have been reported to be associated with CRPS. Age has been considered as a consistent potential risk factor for the onset of CRPS, and higher CRPS risk was correlated with older age [51, 52]. Female sex (particularly postmenopausal) poses a higher risk for the onset of CRPS [53], while the study focused on men in the armed forces by Duman et al. suggests that men 
are also vulnerable [54]. Moreover, it is well established that the time of pain (pre/post-operative or fixation) could also influence the risk of CRPS to some extent. In this study, no significant difference existed in age, gender, or time/onset of pain among patients receiving different treatment methods, which further validated the reliability of the final results to some extent.

Of note, there were several strengths of our study. First, the methods of analysis were systematic and exhaustive. Funnel plots were used to identify potential publication bias because of small-study effects, which have the possibility to result in higher treatment effects compared with large studies. One of the advantages of this network meta-analysis is its ability to use information about specific treatment groups from other trials, and thereby to increase the overall sample sizes. Second, included trials were performed in different regions, which strengthens the external validity. Third, a comprehensive search strategy was used, which assured that all available trial data were selected. Fourth, statistical uncertainty in this network meta-analysis was relatively low, thereby strengthening the confidence in the results.

Nevertheless, these methods also have limitations. First, although all trials were RCTs, most trials were unclear in relevant randomization sequence generations; consequently, selection bias or confounding factors might influence our final results. There was heterogeneity in pooled results, and the source of the difference was often not apparent. Second, grouping treatments that have important differences in rationale and procedures might obscure differences between treatments and cause us to underestimate the relative efficacy of treatment methods. Third, only a limited number of trials ( $n=17$ ) were included in the current network meta-analysis. Consequently, even though the inter-trial heterogeneity was low and could be estimated precisely enough for the primary outcomes of interest, the low number of trials weakened the external validity of our analysis.

In conclusion, the network meta-analysis ranked the seven treatment methods as follows: plaster fixation, non-bridging external fixation, bridging external fixation, dorsal plating, volar plating, dorsal and volar plating and K-wire fixation. Among these methods, we also found that plaster fixation and non-bridging external fixation are more effective in reducing the risk of CRPS in DRF patients, suggesting that plaster fixation and non-bridging external fixation might be better choices for treatment of DRFs.

\section{Acknowledgments}

The authors appreciate the reviewers for their kind collaboration for the sake of improving the quality of this paper.

\section{Conflict of interest}

The authors declare no conflict of interest.

\section{References}

1. Roh YH, BK Lee, Noh JH, et al. Effect of anxiety and catastrophic pain ideation on early recovery after surgery for distal radius fractures. J Hand Surgery 2014; 39: 225864 e2252.

2. Flinkkila T, Sirnio K, Hippi M, et al. Epidemiology and seasonal variation of distal radius fractures in Oulu, Finland. Osteoporosis Int 2011; 22: 2307-12.

3. Shauver MJ, Yin H, Banerjee M, et al. Current and future national costs to medicare for the treatment of distal radius fracture in the elderly. J Hand Surgery 2011; 36: 1282-7.

4. Nellans KW, Kowalski E, Chung KC. The epidemiology of distal radius fractures. Hand Clin 2012; 28: 113-25.

5. Oyen J, Brudvik C, Gjesdal CG, et al. Osteoporosis as a risk factor for distal radial fractures: a case-control study. J Bone Joint Surgery 2011; 93: 348-56.

6. Goehre F, Otto W, Schwan S, et al. Comparison of palmar fixed-angle plate fixation with K-wire fixation of distal radius fractures ( $A O A 2, A 3, C 1)$ in elderly patients. J Hand Surgery 2014; 39: 249-57.

7. Meena S, Sharma P, Gangary SK, et al. Role of vitamin C in prevention of complex regional pain syndrome after distal radius fractures: a meta-analysis. Eur J Orthop Surg Traumatol 2015; 25: 637-41.

8. Marinus J, Moseley GL, Birklein F, et al. Clinical features and pathophysiology of complex regional pain syndrome. Lancet Neurol 2011; 10: 637-48.

9. Kolb L, Lang C, Seifert F, et al. Cognitive correlates of "neglect-like syndrome" in patients with complex regional pain syndrome. Pain 2012; 153: 1063-73.

10. Schneppendahl J, Windolf J, Kaufmann RA. Distal radius fractures: current concepts. J Hand Surgery 2012; 37: 1718-25.

11. Lichtman DM, Bindra RR, Boyer MI, et al. Treatment of distal radius fractures. J Am Acad Orthop Surg 2010; 18: 180-9.

12. Moradi A, Ebrahimzadeh MH, Jupiter JB. Intra-articular fractures of the distal radius: bridging external fixation in slight flexion and ulnar deviation along articular surface instead of radial shaft. Techn Hand Upper Ext Surg 2014; 18: 41-50.

13. Schonnemann JO, Hansen TB, Soballe K. Randomised study of non-bridging external fixation compared with intramedullary fixation of unstable distal radial fractures. J Plast Surg Hand Surg 2011; 45: 232-7.

14. Atroshi I, Brogren E, Larsson GU, et al. Wrist-bridging versus non-bridging external fixation for displaced distal radius fractures: a randomized assessor-blind clinical trial of 38 patients followed for 1 year. Acta Orthop 2006; 77: 445-53.

15. Hsu LP, Schwartz EG, Kalainov DM, et al. Complications of $\mathrm{K}$-wire fixation in procedures involving the hand and wrist. J Hand Surg 2011; 36: 610-6.

16. Wenjing L, Fang D. Care of plaster immobilization. Value Engineering 2011; 36: 254.

17. Wei J, Yang TB, Luo W, et al. Complications following dorsal versus volar plate fixation of distal radius fracture: a meta-analysis. J Intern Med Res 2013; 41: 265-75.

18. Johnson NA, Cutler L, Dias JJ, et al. Complications after volar locking plate fixation of distal radius fractures. Injury 2014; 45: 528-33. 
19. Windolf $M$, Schwieger K, Ockert B, et al. A novel nonbridging external fixator construct versus volar angular stable plating for the fixation of intra-articular fractures of the distal radius: a biomechanical study. Injury 2010; 41: 204-9.

20. Dzaja I, MacDermid JC, Roth J, et al. Functional outcomes and cost estimation for extra-articular and simple intra-articular distal radius fractures treated with open reduction and internal fixation versus closed reduction and percutaneous Kirschner wire fixation. Can J Surg 2013; 56: 378-84.

21. Sutton A, Ades AE, Cooper N, et al. Use of indirect and mixed treatment comparisons for technology assessment. Pharmacoeconomics 2008; 26: 753-67.

22. Liao WC, Chien KL, Lin YL, et al. Adjuvant treatments for resected pancreatic adenocarcinoma: a systematic review and network meta-analysis. Lancet Oncol 2013; 14: 1095-103

23. Higgins JP, Altman DG, Gotzsche PC, et al. The Cochrane Collaboration's tool for assessing risk of bias in randomised trials. BMJ 2011; 343: d5928.

24. Chen $H$, Manning AK, Dupuis J. A method of moments estimator for random effect multivariate meta-analysis. Biometrics 2012; 68: 1278-84

25. Jackson D, White IR, Riley RD. Quantifying the impact of between-study heterogeneity in multivariate metaanalyses. Statistics in Medicine 2012; 31: 3805-20.

26. Peters JL, Sutton AJ, Jones DR, et al. Comparison of two methods to detect publication bias in meta-analysis. JAMA 2006; 295: 676-80.

27. Chaimani A, Higgins JP, Mavridis D, et al. Graphical tools for network meta-analysis in STATA. PloS One 2013; 8 e76654.

28. Song F, Altman DG, Glenny AM, et al. Validity of indirect comparison for estimating efficacy of competing interventions: empirical evidence from published meta-analyses. BMJ 2003; 326: 472

29. Egger M, Davey Smith G, Schneider M, et al. Bias in meta-analysis detected by a simple, graphical test. BMJ 1997; 315: 629-34.

30. White IR, Barrett JK, Jackson D, et al. Consistency and inconsistency in network meta-analysis: model estimation using multivariate meta-regression. Res Synth Methods 2012; 3: 111-25.

31. Abramo A, Kopylov P, Geijer M, et al. Open reduction and internal fixation compared to closed reduction and external fixation in distal radial fractures: a randomized study of 50 patients. Acta Orthop 2009; 80: 478-85.

32. Aktekin CN, Altay M, Gursoy Z, et al. Comparison between external fixation and cast treatment in the management of distal radius fractures in patients aged 65 years and older. J Hand Surg 2010; 35: 736-42.

33. Grewal R, MacDermid JC, King GJ, et al. Open reduction internal fixation versus percutaneous pinning with external fixation of distal radius fractures: a prospective, randomized clinical trial. J Hand Surg 2011; 36: 1899-906.

34. Grewal R, Perey B, Wilmink $M$, et al. A randomized prospective study on the treatment of intra-articular distal radius fractures: open reduction and internal fixation with dorsal plating versus mini open reduction, percutaneous fixation, and external fixation. J Hand Surg 2005 30: 764-72.

35. Hayes AJ, Duffy PJ, McQueen MM. Bridging and non-bridging external fixation in the treatment of unstable fractures of the distal radius: a retrospective study of 588 patients. Acta Orthop 2008; 79: 540-7.

36. Jakubietz MG, Gruenert JG, Jakubietz RG. Palmar and dorsal fixed-angle plates in AO C-type fractures of the distal radius: is there an advantage of palmar plates in the long term? J Orthop Surg Res 2012; 7: 8.

37. Kreder HJ, Hanel DP, Agel J, et al. Indirect reduction and percutaneous fixation versus open reduction and internal fixation for displaced intra-articular fractures of the distal radius: a randomised, controlled trial. J Bone Joint Surg 2005; 87: 829-36.

38. Krishnan J, Wigg AE, Walker RW, et al. Intra-articular fractures of the distal radius: a prospective randomised controlled trial comparing static bridging and dynamic non-bridging external fixation. J Hand Surg 2003; 28: 417-21.

39. McQueen MM. Redisplaced unstable fractures of the distal radius. A randomised, prospective study of bridging versus non-bridging external fixation. J Bone Joint Surg 1998; 80: 665-9.

40. McQueen MM, Hajducka C, Court-Brown CM. Redisplaced unstable fractures of the distal radius: a prospective randomised comparison of four methods of treatment. J Bone Joint Surg 1996; 78: 404-9.

41. Westphal T, Piatek S, Schubert S, et al. Outcome after surgery of distal radius fractures: no differences between external fixation and ORIF. Arch Orthop Trauma Surg 2005; 125: 507-14.

42. Wilcke MK, Abbaszadegan H, Adolphson PY. Wrist function recovers more rapidly after volar locked plating than after external fixation but the outcomes are similar after 1 year. Acta Orthop 2011; 82: 76-81.

43. Wong TC, Chiu Y, Tsang WL, et al. Casting versus percutaneous pinning for extra-articular fractures of the distal radius in an elderly Chinese population: a prospective randomised controlled trial. J Hand Surg 2010; 35: 202-8.

44. Wright TW, Horodyski M, Smith DW. Functional outcome of unstable distal radius fractures: ORIF with a volar fixed-angle tine plate versus external fixation. J Hand Surg 2005; 30: 289-99.

45. Xu GG, Chan SP, Puhaindran ME, et al. Prospective randomised study of intra-articular fractures of the distal radius: comparison between external fixation and plate fixation. Ann Acad Med Singapore 2009; 38: 600-6.

46. Yu YR, Makhni MC, Tabrizi S, et al. Complications of low-profile dorsal versus volar locking plates in the distal radius: a comparative study. J Hand Surg 2011; 36: 1135-41.

47. Salanti G, Ades AE, Ioannidis JP. Graphical methods and numerical summaries for presenting results from multiple-treatment meta-analysis: an overview and tutorial. J Clin Epidemiol 2011; 64: 163-71.

48. DiDomenico LA, Sann P. Univalve split plaster cast for postoperative immobilization in foot and ankle surgery. J Foot Ankle Surg 2013; 52: 260-2.

49. Sugiyama Y, Naito K, Igeta Y, et al. Treatment strategy for distal radius fractures with ipsilateral arteriovenous shunts. J Hand Surg 2014; 39: 2265-8.

50. Potestio D, Laurenti F, Braidotti P, et al. Distal radial fracture: preliminary results with a new non-bridging external fixation technique. J Bone Joint Surg 2011; 93: 188-8.

51. Jellad A, Salah S, Ben Salah Frih Z. Complex regional pain syndrome type I: incidence and risk factors in patients with fracture of the distal radius. Arch Phys Med Rehabil 2014; 95: 487-92.

52. Moseley GL, Herbert RD, Parsons T, et al. Intense pain soon after wrist fracture strongly predicts who will develop complex regional pain syndrome: prospective cohort study. J Pain 2014; 15: 16-23. 
53. Pons T, Shipton EA, Williman J, et al. Potential risk factors for the onset of complex regional pain syndrome type 1: a systematic literature review. Anesthesiol Res Pract 2015; 2015: 956539.

54. Duman I, Dincer U, Taskaynatan MA, et al. Reflex sympathetic dystrophy: a retrospective epidemiological study of 168 patients. Clin Rheumatol 2007; 26: 1433-7. 